Geopolítica(s) Revista de estudios sobre espacio y poder ISSN: 2172-3958

\title{
Comentario. La imaginación geopolítica (amazónica) de Bertha Becker $^{1}$
}

Fabio Candotti ${ }^{2}$ e Flávia Melo ${ }^{3}$

Recibido: 5 de abril de 2018 / Aceptado: 10 de septiembre de 2018

Sumario. Introducción. 1. Una geopolítica ambivalente. 2. Saudades de Bertha. Bibliografía.

Cómo citar: Candotti, Fabio \& Melo, Flávia (2019) "Comentario. La imaginación geopolítica (amazónica) de Bertha Becker". Geopolítica(s). Revista de estudios sobre espacio y poder, vol. 10, núm. 1, 153-159.

\section{Introducción}

A lo largo de los siglos, la Amazonía ha inspirado un conjunto amplio y diversificado de "imaginaciones geopolíticas" —en el sentido más amplio atribuido por Agnew (2005) - . Desde su "invención" en los primeros tiempos de la colonización (Gondin, 2007) y las disputas ibéricas por su territorio (González, 2014), hasta sus recientes "metamorfosis" (Silva, 2000) y su "ambientalización" (Santos, 2014). En las últimas décadas del siglo XX y en las primeras del XXI, fue objeto de diversas consideraciones "geopolíticas" en América del Sur, delimitadas por territorios estatales, como es el caso de Colombia (Van Vliet, 1990), en Perú (IIAP, 2009) y en Bolivia (García Linera, 2013) .

El artículo que presentamos es una expresión de esas imaginaciones geopolíticas más recientes sobre la Amazonía del siglo XXI. Se trata de una conferencia, pronunciada en 2004 y publicada en 2005, de una de las más influyentes geógrafas brasileñas de los últimos 40 años: Bertha Koiffmann Becker (1930-2013). En aquel momento, sus contribuciones estaban vigorosamente centradas en el campo de las políticas públicas y su texto expresa tanto la madurez de su pensamiento como un nuevo momento de su compromiso con los proyectos de Estado para la Amazonía. Formada en Geografía e Historia en 1952 y profesora en la Universidad Federal de Rio de Janeiro de 1958 hasta su muerte, Becker dedicó casi toda su carrera profe-

1 (Nota de la redacción) Comentario sobre el texto de Bertha K. Becker (2005) "Geopolítica da Amazônia".

Estudos Avançados, vol. 19, núm. 53, 71-86.

2 Departamento de Ciencias Sociales, Universidade Federal del Amazonas (Brasil).

E-mail: fmcandotti@gmail.com

3 Programa de Posgrado en Antropología Social, Universidade de São Paulo (Brasil).

E-mail: flaviamelodacunha@usp.br 
sional al estudio de esa región, marcado por un esfuerzo incansable de elaboración de propuestas para su transformación.

Desde las primeras expediciones junto a sus estudiantes de diplomacia brasileña en los años 1960, hasta su largo protagonismo en las políticas de los gobiernos del Partido de los Trabajadores, entre 2003 y 2013, el listado de su participación en instituciones del Estado y de la sociedad civil es impresionante. Lo que no sería tan particular en la historia de la geografía si, en medio de los conocidos límites del mundo de las políticas públicas, Becker no hubiese desarrollado una interpretación teóricamente compleja y poderosa de la Amazonía junto a una concepción singular de la geopolítica, cuya ambivalencia deseamos discutir.

Becker empieza sus investigaciones amazónicas en los años 1970 en medio de un conjunto más amplio de estudios sobre las fronteras móviles de explotación capitalista (Velho, 1976; Martins, 1975). La gran extensión de sus expediciones de campo, persiguiendo la construcción de carreteras del estado del Acre hasta el estado de Pará (Becker, 1974, 1976, 1977) ampliaron su percepción de la región. Así, en un contexto económico de expansión agrícola y en medio de una lenta transición de la dictadura militar (1964-1985) a la democracia, surge un nuevo diagnóstico y, también, una nueva cuestión teórica (para la geografía) y política (para la democracia): la urbanización de la Amazonía. Un tema que le ha acompañado desde su primero artículo (1974) hasta su último libro, A Urbe Amazônida (2013).

\section{Una geopolítica ambivalente}

En ese primer momento, en diálogo con Harvey (1973) y Lefebvre (1978) y, por lo tanto, a través de una caja de herramientas marxista, Becker (1985) propuso una interpretación de la urbanización como "la otra cara" de la frontera - concepto que, siguiendo a Velho (1976), se definía como "un espacio no plenamente estructurado y, como tal, generador de realidades nuevas y dotado de elevado potencial político" (Becker 1985: 385) - . En ese espacio lleno de potencias, los "núcleos urbanos" asumían, para ella, un papel "generativo" (Becker, 1985: 369) contradictorio: por un lado, como partes de la cadena de extracción de excedente a través de la explotación de la movilidad de la fuerza de trabajo, del capital financiero y de la política ideológica del Estado; por otro lado, como espacios de conflictos sociales intensos. Con base en ese diagnóstico, en el año en que oficialmente terminaba la dictadura militar, Becker se preguntaba:

En vista del papel generativo que lo urbano puede tener, la mayor facilidad de asignación de inversiones que ofrece, y teniendo en cuenta que el poder local es la parte más vulnerable del aparato de Estado, y que puede estar sujeto a presiones locales, ¿no sería lícito considerar la urbanización, junto con la distribución de tierras y reforma agraria, una alternativa social y políticamente válida para ser mejor analizada y utilizada? (Becker 1985: 370)

Esa contradicción económica y política de lo urbano en la Amazonía —entre la explotación y la potencialidad emancipadora - se convertía en el centro de su crítica a la geopolítica de los militares, así como de su propia construcción de una nue- 
va perspectiva geopolítica para la democracia. Ese doble movimiento teórico se queda evidente en un importante y largo artículo publicado en 1988, "La Geografía y el rescate de la Geopolítica", donde se presenta una nueva caja de herramientas (Sack, 1986; Raffestin, 1980; Foucault, 1978). Por eso, Becker fue incluida entre las/os "exponentes del movimiento de renovación del pensamiento geográfico brasileiro" (Vieira et al., 2014: s/p).

Con esa nueva mirada, en el mismo año de la nueva Constitución de Brasil (1988), Becker analiza cuidadosamente la geopolítica de los militares, empezando por su concepción teórica hasta su puesta en práctica durante la dictadura militar. Becker demuestra la coherencia entre el "proyecto geopolítico de la modernidad" (Becker, 1988: 133) del General Golbery do Couto e Silva (1967) y la política militar de los años 1970, sobre todo por la centralidad del "vector científicotecnológico moderno" (Becker, 1988: 139) en la producción del espacio nacional. Una "Geopolítica Nacional" (Becker, 1988: 136) autoritaria en la cual la ocupación de la Amazonía es uno de los objetivos centrales - y explícitamente diseñado en el Programa de Integración Nacional de 1970 (Decreto-ley n. 1106/1970)—. Para la geógrafa, la consecuencia de eso proceso fue la superposición conflictiva entre una "malla programada" (o "proyectada") y una "malla sociopolítica viva", siendo los "nuevos movimientos sociales" un "síntoma" de esa relación (Becker, 1988: 142).

Es a través de esa misma clave de análisis, que, en el mismo artículo, Becker presenta sus "cuestiones para la reconstrucción de la Geopolítica en el fin del siglo XX" (Becker, 1988: 119). En ese punto, la ambivalencia de su perspectiva geográfica se muestra con mucha fuerza. Primero, su movimiento de "rescate" vuelve a Ratzel (1987), considerando la Geopolítica (siempre con G mayúscula) un "saber estratégico" (Becker, 1988: 117) en tiempos marcados por la emergencia, a partir de la postguerra, de una "Cronopolítica" (Virilio \& Lotringer, 1983), asociada a un "orden mundial militarizado" (Becker, 1988: 120), y al poder de las corporaciones económico-financieras. La ausencia de ese saber, para ella, "constituye una pérdida de poder y un impedimento a la gestión democrática del territorio" (Becker, 1988: 117). Hay en ello una cierta concepción pragmática, que concibe ese saber como "una expresión y un instrumento de las relaciones de poder actuantes en la producción del complejo espacio global contemporáneo" (Becker, 1988: 144). Al mismo tiempo, Becker anuncia que:

La Geopolítica que queremos rescatar es la del reconocimiento sin fetichización de la potencialidad política y social del espacio, o sea, la del saber sobre las relaciones entre espacio y poder. Poder multidimensional, derivado de múltiples fuentes, inherente a todos los actores, relación social presente en todos los niveles espaciales. Espacio, dimensión material, constituyente de las relaciones sociales y, por eso mismo, siendo, en sí mismo, un poder (Becker, 1988: 119).

En resumen, Becker propone una composición original entre Ratzel y Raffestin. Esa concepción ambivalente de la geopolítica ${ }^{4}$ se hace presente en la conferencia

$4 \quad$ Al apuntar esa ambivalencia, nos alejamos de la interpretación de Vieira et al. (2014) que, a pesar de también percibir en la obra de Becker un medio camino "ni tanto al cielo, ni tanto a la tierra" (Vieira et al. 2014: s/p), entienden que hay, por un lado, una geopolítica (como dominio de las "acciones y estrategias de los Estados"), y, por otro, una geografía política, (atenta a los "diferentes actores sociales en la conformación territorial") 
de 2004 cuya traducción publicamos. Realizada en el Instituto de Estudios Avanzados de la Universidad de São Paulo y publicada un año después con un título homónimo a su primer libro (Becker, 1982) ${ }^{5}$, esa conferencia se sitúa en un nuevo contexto histórico, compuesto por nuevas imaginaciones geopolíticas sobre la Amazonía. Mas allá del conocido surgimiento (transnacionalizado) de la cuestión ambiental en Brasil, aquel momento era, para muchos, de optimismo en cuanto a las políticas de Estado, con la llegada reciente del Partido de los Trabajadores al gobierno federal (2003). Un nuevo horizonte político compartido por Becker, que ya tenía papel activo en el Grupo de Trabajo Amazónico ${ }^{6}$ y que trabajaba junto a Marina Silva en el Ministerio del Medio Ambiente para la elaboración del Plan Amazonía Sostenible.

Esa nueva situación de la política del Estado — subentendida en la conferencia - se suma a otros cambios en el contexto histórico, presentados detalladamente como suelo para un nuevo saber estratégico geopolítico para la Amazonía brasileña. Un verdadero proyecto de cambio del "patrón de desarrollo" (Becker, 2005: 72) para un espacio con un nuevo lugar en el "sistema espacial nacional" (Becker, 2005: 82): una "región en sî", con "estructura productiva propia y múltiples proyectos de diferentes actores" (Becker, 2005: 80) (como gobiernos, empresas, universidades y movimientos sociales internacionalizados). Una propuesta no solamente optimista como profundamente pragmática, que intentaba forjar un consenso, muy improbable, entre actores sociales organizados que "tienen sus propias territorialidades, por encima y por debajo de la escala del Estado, y sus propias geopolíticas" (Becker, 2005: 72). Por un lado, delante de las arcaicas fuerzas de explotación de recursos naturales, defendía una lógica "civilizatoria y cultural" (Becker, 2005: 74) de inspiración ambientalista y la lucha de las poblaciones tradicionales, subrayando incluso la importancia de sus saberes. Por otro lado, comprendía que la urbanización de la región era incontenible - confirmando la idea anterior de uno "bosque urbanizado" (Becker, 1995) - Confrontando el discurso crítico de los estudiosos sobre el "arco de fuego" (Becker, 2005: 81) que avanzaba entre el sur y el leste de la región amazónica, Becker defendía que la frontera móvil de explotación de recursos se habría convertido en un "arco de poblamiento consolidado" (Becker, 2005: 83).

A través de esa posición diplomática, Becker se plantea el desafío de "superar la polaridad conflictiva entre la política ambiental y la de desarrollo" (Becker, 2005: 83) y "encontrar modos de compatibilizar" (Becker, 2005: 72), en la Amazonía brasileña, tres de las palabras de orden más importantes de las artes contemporáneas de gobierno: el "crecimiento económico, la conservación de los recursos naturales y la inclusión social" (Becker, 2005: 72). Entre otras propuestas más convencionales que contenían incluso una nueva "ordenación del territorio" (Becker,

(Vieira et al. 2014: s/p). Se trata de una interpretación posible, pero preferimos tomar en serio la opción de Becker en conceptualizar la geopolítica en diálogo con Sack, Raffestin e Foucault.

5 Compilación de artículos escritos entre 1972 y 1981, aún en el marco de sus estudios críticos sobre fronteras de expansión. El libro, sin embargo, anticipa diversos temas y construcciones teóricas centrales en sus trabajos de las décadas siguientes, como su análisis del "bosque urbanizado" (Becker, 1982: 122-123), o la "urbe amazónica”, una atención a la fragilidad ecológica de la región y, aún, un cierto esbozo de su perspectiva geopolítica. Para una detallada presentación del libro, ver Melo (1983).

6 Creado en 1992, el GTA consiste en una red de más de 600 entidades de la sociedad civil que actúa en el monitoreo de políticas y de conflictos socio ambientales para la sostenibilidad de la biodiversidad amazónica. 
2005: 83), la más audaz fue la convergencia entre el conservacionismo de los movimientos socioambientales y la nueva "frontera" (Becker, 2005: 74) del capitalismo informacional. Becker proponía la valorización del capital natural, o sea, la transformación de la biodiversidad en mercancía mediada por la biotecnología. Solución que, según la geógrafa, permitiría al bosque tener un "valor económico para competir con la madera, con la ganadería y con la soja" (Becker, 2005: 85), una vez que, para esa industria lo más valioso no son las cantidades de recursos materiales si no la diversidad inmaterial de informaciones genéticas.

La ambivalencia en el concepto de geopolítica de Becker, presente desde los años 1980, permanece en la conferencia de 2004. Pero, también es evidente cómo uno de los sentidos gana más densidad y hace gravitar al otro a su alrededor. La mirada crítica hacia las relaciones difusas entre espacio y poder se expresa a través de una atención a las "territorialidades" (Becker, 2005: 72) que, mientras tanto, se sujetan cada vez más a la gestión estatal (Becker, 2010). Al mismo tiempo, la defensa de la geopolítica como un saber estratégico - en el doble sentido del término- es tan central que se proyecta sobre los actores sociales. Así que la Geopolítica de la Amazonia se expresa como una tentativa de síntesis - no tan dialéctica cuanto diplomáticamente democrática- de diferentes estrategias geopolíticas. Entre tanto, al fin, actualiza la imaginación geopolítica militar, concediendo nueva centralidad al vector técnico-científico.

Así como en el texto ahora traducido, la extensa obra de Becker sobre la Amazonía - compilada recientemente en tres volúmenes (Becker, 2015) - parece expresar las contradicciones del encuentro de la Geografía Política con el Estado en una de sus versiones contemporáneas, marcada por un frágil régimen democrático liberal y atravesada por una racionalidad neoliberal —incluso de tipo "social" o "de izquierda" (Dardot \& Laval, 2009) — que hace del mercado la única vía posible de superación de los efectos perversos del propio capitalismo.

\section{Saudades de Bertha}

En los últimos quince años, las influencias de Becker son perceptibles en la formulación de una serie inédita de políticas para el desarrollo amazónico, que pusieron énfasis a la inclusión social de las poblaciones urbanas y tradicionales y, principalmente, al ordenamiento territorial. Un buen ejemplo fue la construcción del programa Territorios de la Ciudadanía ${ }^{7}$, orientado al desarrollo territorial y humano, e involucrando conocimientos tradicionales y la explotación de recursos de la biodiversidad (Brasil 2008).

Estas políticas fueron diseñadas y gestadas al mismo tiempo en que se fortalecían espacios de actuación de la sociedad civil y movimientos sociales, que Becker reconocía como actores emergentes y centrales para el desarrollo de la región (Becker, 2005: 71). Añadimos a ello el crecimiento, bastante impresionante, de las instituciones de investigación y educación, del fomento para proyectos y redes de investigación y la proliferación de grupos de investigación con sede en la región

Estrategia de universalización de acciones para la ciudadanía implementada en regiones del país consideradas como “más vulnerables" (Brasil, 2008: 2). 
Norte del país. Transformación de la que hemos sido testigos a lo largo de nuestras carreras de investigación y docencia.

Sin embargo, como Becker pronunciaba en las primeras líneas del artículo traducido en esta edición, un elemento fue decisivo para la frustración de ese proyecto de desarrollo de la Amazonía. A pesar de sus propósitos, de la pertinencia y de la aceptación de sus argumentos por segmentos del Estado brasileño, las políticas efectivamente implementadas (con mayor o menor éxito) fueron insuficientes para descentralizar el dominio tecnológico, superar las desigualdades regionales (internas y externas) y contener el avance de la explotación predatoria del bosque. Tampoco logró promover la ampliación del uso de los avances científicos y tecnológicos en favor de ese proyecto. Es decir, las "redes" no se "socializaron" con la intensidad y extensión esperada por Becker en 2004 (Becker, 2005: 71).

Aunque muy reciente, la transición en el gobierno brasileño sugiere cambios significativos en lo que se refiere a la geopolítica de la Amazonía, en los términos de Becker. Para algunos, presenciamos el resurgimiento de la "economía de frontera" (Becker, 2005: 72) ${ }^{8}$, paradigma del desarrollo amazónico y latinoamericano que Becker tanto combatió. En este nuevo e incierto escenario, el intento de construir un consenso democrático entre las perspectivas socioambiental y desarrollista, apoyado en el sueño de una cadena de producción biotecnológica parece huir, y las Amazônias de Becker, de las poblaciones tradicionales y urbanas, de los ambientalistas y de tantos científicos parecen encontrarse bajo una amenaza de grandeza desconocida.

Más que nunca, sentimos saudades de la imaginación geopolítica de Becker, con toda su ambivalencia.

\section{Bibliografía}

Agnew, John (2005) Geopolítica: una re-visión de la política mundial. (Trad. María Lois.) Madrid: Trama Editorial.

Becker, Bertha K. (1974) “A Amazonia na Estrutura Espacial do Brasil”. Revista Brasileira de Geografia, vol. 36, núm. 2, 3-33.

Becker, Bertha K. (1976) "Uma hipótese sobre a origem do fenômeno urbano numa fronteira de recursos do Brasil". Revista Brasileira de Geografia, IBGE, Rio de Janeiro, vol. 40, núm. 1, 111-22.

Becker, Bertha K. (1977) “A implantação da rodovia Belém-Brasília e o desenvolvimento regional". Anuário do Instituto de Geociências, UFRJ, Rio de Janeiro, 156 p.

Becker, Bertha K. (1982) Geopolitica da Amazonia: A Nova Fronteira de Recursos. Rio de Janeiro: Zahar.

Becker, Bertha K. (1985) "Fronteira e urbanização repensadas". Revista Brasileira de Geografia, Rio de Janeiro, 47 (3/4).

Becker, Bertha K. (1988). "A Geografia e o Resgate da Geopolítica”. Revista Brasileira de Geografia, vol. 50, núm. 2 (especial), 99-125.

Becker, Bertha K. (1995) "Undoing Myths: The Amazonan - an Urbanized Forest" en M. Clüsener-Godt \& I. Sachs. (eds). Brasilian perspectives on Sustainable Development of the Amazon Region. Paris: UNESCO/Parthenon, 53-89.

8 Becker menciona el concepto de "economía de frontera" de Kenneth Boulding (1966). 
Becker, Bertha K. (2010) "Novas territorialidades na Amazônia: desafio às políticas públicas”. Boletim do Museu Paraense Emílio Goeldi. Ciencias Humanas, vol. 5, núm. 1, 17 23.

Becker, Bertha K. (2013) A urbe amazônida: a floresta e a cidade. Rio de Janeiro, RJ: Garamond, 2013.

Becker, Bertha K. (2015) As Amazônias de Bertha Becker: ensaios sobre geografia e sociedade na região amazônica. Vol. 1, 2 e 3. Org. Ima Célia Guimarães Vieira. Rio de Janeiro: Garamond.

Boulding, Keneth (1966) “The Economics of Coming Space-ship Earth" en: Environment Quality in a Growing Economy. Baltimore: John Hopkins.

Brasil (2008) Programa Territórios da Cidadania. Brasília/DF: Presidência da República.

Couto e Silva, Golbery do (1967) Geopolítica do Brasil. Rio de Janeiro: José Olympio.

Dardot, Pierre \& Laval, Christian (2009) La nouvelle raison du monde. Essai sur la société néolibérale. Paris: La Découverte.

Foucault, Michel (1978) Microfísica Del Poder. Genealogía Del Poder. (Ed. y Trad. de Julia Varela y Fernando Álvarez-Uría. Madrid: La Piqueta.

Gondim, Neide (2007) A Invenção da Amazônia. $2^{\mathrm{a}}$ Ed. Editora Valer. Manaus.

González, Sebastián G. (2014) Frontera Salvática: españoles, portugueses y su disputa por el noroccidente amazónico, siglo XVIII. Bogotá: Instituto Colombiano de Antropología e Historia - ICANH.

Harvey, David (1973). Social justice and the city. Londres: Edward Arnold.

IIAP (2009) Amazonía Peruana: visión de desarrollo, potencialidades y desafíos. Iquitos: Instituto de Investigaciones de la Amazonía Peruana.

Lefebvre, Henri (1978) De l'Etat. Paris: Union Génerale.

García Linera, Álvaro (2013) Geopolítica de la Amazonía: poder hacendal-patrimonial y acumulación capitalista. La Paz: Vicepresidencia del Estado Plurinacional de Bolívia.

Martins, José de Souza (1975) Capitalismo e tradicionalismo. São Paulo: Livraria Pioneira Editora.

Melo, Mário Larceda de (1983) "Resenha de Geopolítica da Amazônia, de Bertha Becker". Ciência \& Trópico, vol. 11, núm. 1, 135-138.

Oliveira, Antônio P. (2019) Polo Industrial de Manaus: a biodiversidade como mercadoria. mimeo.

Raffestin, Claude (1980) Pour une Géographie du pouvoir. Paris: Ed. Litec.

Ratzel, Friedrich (1987) La Géographie politique. Les concepts foudamentaux. Paris: Ed. Fayard.

Sack, Robert D. (1986) Human Territoriality: its Theory and History. Cambridge: Cambridge University Press.

Santos, Luiz Fernando S. (2014) O panóptico verde: a invenção ambiental da Amazônia. Manaus: Editora Valer/FAPEAM.

Silva, Marilene Corrêa da (2000) Metamorfoses da Amazônia. Manaus: EDUA.

Van Vliet, Klaas Geert (1990) "Reflexiones sobre geopolítica en la Amazonia Colombiana" en coordinadores K. G. van Vliet, J. J. Vieco, M. Useche (eds) Amazonia, identidad y desarrollo. Bogotá: Fundación Manoa y FEN Colombia.

Velho, Otávio G. (1976) Capitalismo autoritário e campesinato. Rio de Janeiro: Zahar.

Vieira, Ima Célia G.; Toledo, Peter M.; Rocha, Gilberto \& Santos Jr, Roberto A. (2014) "Bertha Becker e a Amazônia". [Puesto en línea en 25 de diciembre de 2014. URL: $<$ http://www.ub.edu/geocrit/b3w-1103-4.htm>. Consultado el 4 de marzo de 2019].

Virilio, Paul \& Lotringer, Sylvère (1983) Pure War. Cambridge, MA: Semiotext[e]/MIT Press. 